\title{
Cooked Green Banana in Hospitalized Children With Acute Watery Diarrhea Without Dehydration
}

\author{
Mohammod Jobayer Chisti and Monira Sarmin \\ Dhaka Hospital, Nutrition and Clinical Services Division, International Centre for Diarrhoeal Disease Research, Bangladesh \\ (icddr, b), Dhaka, Bangladesh. chisti@icddrb.org
}

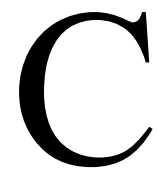

hildhood diarrhea is one of the leading health problems, especially in low and middleincome countries (LMICs), and accounts for $8 \%$ of the annual 5.2 million under-five global deaths $[1,2]$. Almost two-third of all under-five diarrheal deaths are reported in sub-Saharan Africa and South-East Asia [1]. Although, most of the LMICs including India failed to achieve the Millennium Development Goals (MDGs), especially the Goal-4 that aimed to reduce under-five child mortality including the deaths from diarrhea; these countries have now targeted to achieve the Sustainable Development Goals (SDGs). The SDG-3 specifically targets to reduce under-five mortality at least by 25 per 1,000 live births [3] and therefore more emphasis should be given for the reduction of diarrhea related under-five deaths. Prompt and adequate management of diarrhea is the principal factor of targeted approach for reduction of diarrheal deaths [4]. World Health Organization (WHO) recommended oral rehydration solution (ORS) and oral zinc therapy in addition to frequent breast feeding, and home available fluids are the mainstay of management of diarrhea [5]. Although, ORS is credited with saving millions of lives by correcting dehydration, it does not have any impact on the reduction of duration of diarrhea. However, zinc is already shown to have reduction of diarrheal duration and hospitalization [6].

In this issue of Indian Pediatrics, Gunasekaran and colleagues [7] share their findings from a randomized controlled trial (RCT) on the efficacy of green banana (Musa paradisiaca) for recovery in children ( 9 months to 5 years) with acute watery diarrhea with no dehydration. Children in the control group $(n=125)$ received standard care, and children in the intervention group $(n=125)$ received cooked green banana in addition to standard care. Patients were hospitalized during the initial 72 hours and thereafter continued treatment at home until diarrhea stopped or the 14th day of illness, whichever was earlier. A significantly higher proportion of children recovered in the green banana group compared to the control group (62.4\% vs. $47.2 \% ; P=0.002)$ and there was $85 \%$ lowered risk of dehydration and $70 \%$ lowered risk of developing persistent diarrhea in the green banana group compared to their counterparts. The entry criteria were a bit specific as the study included children with acute watery diarrhea (AWD) without any degree of dehydration defined by the WHO and excluded children who were undernourished (weight for age $Z$ score $<-2$ ), and this limits the generalizability of the study. This RCT happens to be a hospital-based study although the WHO recommends that children with AWD without dehydration or under-nutrition or any co-morbidity need to be treated at home. Gunasekaran, et al. [7] also did not mention the number of children who were admitted with AWD during the study period and how many of them had dehydration. The reasons for the non-receipt of allocated interventions in 17 children in the green banana group were not specified in the CONSORT flow diagram. It is difficult to understand whether the supportive care at home for both the groups were similar. However, despite these limitations, this RCT revealed some valuable findings that underscored the benefit of the use of cooked green banana for the treatment of children with AWD without having any form of dehydration and undernutrition in developing countries.

Previously, a community-based study from Bangladesh [8] evaluated the beneficial role of green banana in children not only having AWD but also with prolonged diarrhea. Gunasekaran and his colleagues conducted this trial in the hospital and reemphasized the importance of using green banana in faster recovery of childhood AWD [7]. Their data highlighted the role of cooked green banana supplemented diet as a useful adjunct to standard treat-ment (ORS, zinc, and home available fluids) in the management of AWD with no dehydration and no undernutrition.

Overall, Gunasekaran and colleagues should be congratulated for this important work. Importantly, we need to be cautious in undertaking the problem of AWD 
in developing countries, which may require an unbiased approach relating to clinical care with basic public health measures including provision of clean water, sanitation, nutrition, and immunization. In LMICs, these should be the cornerstones of any efforts to reduce the incidence of diarrhea as well as deaths from diarrhea, and this in turn may help to achieve SDG-3 by reducing under-five mortality by two-thirds till 2030.

Funding: Nil; Competing interests: None stated.

\section{REFERENCES}

1. UNICEF. Levels and trends in child mortality: report 2019. Estimates developed by the UN Inter-agency Group for child mortality estimation. 2019.

2. Kotloff KL, Nataro JP, Blackwelder WC, et al. Burden and aetiology of diarrhoeal disease in infants and young children in developing countries (the Global Enteric Multicenter Study, GEMS): a prospective, case-control study. Lancet. 2013;382:209-22.

3. World Health Organization. World health statistics 2020: monitoring health for the SDGs, sustainable development goal. 2020.

4. Dhingra U, Kisenge R, Sudfeld CR, et al. Lower-dose zinc for childhood diarrhea - A randomized, multicenter trial. $\mathrm{N}$ Engl J Med. 2020;383:1231-41.

5. World Health Organization. Pocket Book for Hospital Care of Children: Guidelines for the Management of Common Illness with Limited Resource. World Health Organization, 2013.

6. Black RE. Progress in the use of ORS and zinc for the treatment of childhood diarrhea. J Glob Health. 2019; 9:010101.

7. Gunasekaran D, Chandramohan A, Karthikeyan K, Balasubramaniam B, Jagadeesan P, Soundararajan P. Effect of green banana (Musa paradisiaca) on recovery in children with acute watery diarrhea with no dehydration - A randomized controlled trial. Indian Pediatr. 2020;57:1114-18.

8. Rabbani GH, Larson CP, Islam R, Saha UR, Kabir A. Green banana-supplemented diet in the home management of acute and prolonged diarrhoea in children: A community-based trial in rural Bangladesh. Trop Med Int Health. 2010;15:1132-9. 